\title{
Scheduling Wireless Links with Successive Interference Cancellation
}

\author{
Olga Goussevskaia \\ Department of Computer Science \\ Federal University of Minas Gerais, Brazil \\ Email: olga@dcc.ufmg.br
}

\author{
Roger Wattenhofer \\ Computer Engineering and Networks Laboratory \\ ETH Zurich, Switzerland \\ Email: wattenhofer@tik.ee.ethz.ch
}

\begin{abstract}
In this paper we study the problem of scheduling wireless links in a model where successive interference cancellation is combined with the physical interference model and uniform power assignment. Successive interference cancellation is based on the observation that interfering signals should not be treated as random noise, but as well-structured signals. By exploiting this structured nature, the strongest signal can be decoded and subtracted from a collision, thus enabling the decoding of weaker simultaneous signals. The procedure can be repeated iteratively as long as the collided signals differ in strength significantly. It has been shown that the problem of scheduling wireless links with successive interference cancellation is NP-hard. In this work, we propose a polynomial-time scheduling algorithm that uses successive interference cancellation to compute short schedules for network topologies formed by nodes arbitrarily distributed in the Euclidean plane. We prove that the proposed algorithm is correct in the physical interference model and provide simulation results demonstrating the performance of the algorithm in different network topologies. We compare the results to solutions without successive interference cancellation and observe that throughput gains of up to $20 \%$ are obtained in certain scenarios.
\end{abstract}

\section{INTRODUCTION}

The problem of scheduling wireless links is fundamental in determining the communication capacity of a wireless network. Given a set of communication requests between senders and receivers, arbitrarily distributed in space, the problem consists in determining which subsets of links (requests) can be scheduled concurrently, such that no collisions occur among them, and how these subsets must be chosen, such that all links are able to transmit successfully in minimum time.

The link scheduling problem has been studied in a variety of interference models, such as graph-based models (e.g. the protocol interference model) and fading channel models (e.g. the physical interference model). The physical model, to which we also refer as the SINR (Signal to Interference plus Noise Ratio) model, provides a more realistic representation of wireless interference than graph-based models, however, it might be more challenging when it comes to complexity analysis and algorithm design. In [1] it was shown that the link scheduling problem with uniform power assignment is NP-complete in the physical interference model by a reduction from the Integer Partition problem.

In the SINR model, a transmission is considered successful if the signal power of the sender at the intended receiver sufficiently exceeds the sum of signal powers of all concurrent transmissions in the network (considered as interference) at the receiver, i.e., it is assumed that a receiver successfully decodes one, and only one, message at a time. This "traditional" approach of handling wireless interference by avoiding or reducing it is implemented in most of the existing MAC protocols for wireless LANs, which are based on carrier sensing, e.g. 802.11. In carrier sense, a transmission is deferred if the sender device senses another transmission in progress. The idea is to eliminate interference and allow higher SINR at the receiver. The problem with this approach is that it discourages spatial reuse, thus wasting available bandwidth. Since radio devices can typically detect an ongoing transmission over very large areas, a single ongoing transmission can block a great number of potential concurrent transmissions spread over a large area. This basically results in linear scheduling, i.e., one node transmits at a time, whereas many concurrent transmissions could be scheduled simultaneously.

There are alternative models that revise the assumption that interference is necessarily harmful and that a receiver can only decode one strongest signal at a time. Techniques such as cochannel separation, analog network coding, MIMO, and successive interference cancellation have radically changed the definition of a successful transmission. Cochannel separation techniques allow the receiver to decode several signals simultaneously under the assumption that these signals differ significantly in their strength [2]. Analog network coding makes it possible to simultaneously decode two signals of similar strength, under the assumption that the receiver knows one of the interfered signals by having overheard or forwarded it earlier [3]. MIMO systems explore space-time coding techniques by means of antenna arrays and coherent combining at a receiver [4], [5]. In successive interference cancellation, one sender's packet with the strongest signal is first decoded and "subtracted" from the received collision, such that weaker simultaneous signals can be then decoded in an iterative fashion [6].

Given these alternative ways of defining a successful transmission, an interesting question that rises is whether the computational complexity of the scheduling problem remains the same. In [7] it was shown that scheduling wireless links in the SINR model remains NP-hard when two different forms of decoding collided signals are considered: successive 
interference cancellation and analog network coding in a 2way relay topology.

In this work we build upon the results in [7]. We consider the model where successive interference cancellation is combined with the SINR model and uniform power assignment. Successive interference cancellation is based on the observation that interfering signals should not be treated as random noise, but as well structured signals comprised by modulated data. By developing a data-dependent model of the signal, the strongest signal can be decoded and subtracted from the sum of the interfering signals and noise, thus enabling the decoding of the remaining weaker signals. The procedure can be repeated iteratively as long as the collided signals differ in strength significantly.

We propose a polynomial-time scheduling algorithm that uses successive interference cancellation to compute short schedules obeying the SINR constraints for network topologies formed by nodes arbitrarily distributed in the Euclidean plane. We provide simulation results demonstrating the performance of the algorithm in different network topologies. We compare the obtained schedule lengths to solutions that do not employ successive interference cancellation and observe that throughput gains of up to $20 \%$ are obtained in certain scenarios.

In Section II we discuss the related work. In Section III we define the network model that combines SINR and successive interference cancellation constraints. We refer to the scheduling problem defined in this model as Scheduling with Successive Interference Cancellation (SSIC). In Section IV we present the proposed scheduling algorithm for SSIC and the corresponding proof of correctness. In Section V we discuss our simulation results. Finally, in Section VI we present some conclusions.

\section{RELATED WORK}

The problem of scheduling wireless requests with uniform power assignment in the SINR model was shown to be NPcomplete in [1]. After this first hardness result, the analysis of the problem in the physical model has received a lot of attention, generating an interesting body of work. In [8] a constant approximation algorithm was devised for the on-slot scheduling problem under uniform power assignment. More recently, a constant approximation algorithm for the joint problem of scheduling and power control was obtained in [9]. A detailed survey of these and related recent results can be found in [10].

The possibility of decoding collided simultaneous signals has been explored in a variety of contexts. Some techniques, such as cochannel signal separation, explore differences in the characteristics of interfered signals, such as signal's strength, to decode several signals simultaneously [2], [11]. Other techniques, such as MIMO, allow multiple concurrent transmissions using antenna arrays and space-time coding to decode multiple concurrent transmissions simultaneously [5].

Interference cancellation is a type of multi-user detection technique in wireless communication networks [12]. Many of such techniques were originally proposed for CDMA systems [13], [14] and exploit several resources of the cellular infra-structure, such as separate areas of reception and centralized synchronization and power control. In [15] a proof of concept of interference cancellation applied to wireless LANs was presented, where a collision between signals with similar power could be decoded. In [6] a simpler technique called successive interference cancellation was implemented for the 802.15.4 (ZigBee) physical layer. In this approach, collided signals have to differ significantly in power in order to be decoded, and the strongest signal is successively subtracted from a collision, allowing the decoding of weaker simultaneous signals. Successive interference cancellation has been also applied in MIMO LANs: in [16] it was used in combination with interference alignment to improve throughput, and in [4] it was used for channel training and transmission control to avoid coordination and increase throughput.

In [17] Gelal et al. analyzed how topology control can be used to improve the efficiency of successive interference cancellation in MIMO networks. In a more recent work, Avin et al. [18] analyzed the effect of interference cancellation on the topology of "reception zones", i.e., regions where a signal can be successfully decoded in the SINR model.

Another group of related work deals with network coding in the physical layer, or analog network coding (ANC). In [3], [19] ANC algorithms are proposed with the emphasis on decoding two signals that interfered with each other, mainly in the canonical 2-way relay topology. In [20] the concept of ANC was generalized by showing how collided packets can be recovered in a 802.11 network using ZigZag decoding, provided that the network operates at sufficiently high SNR, not too many packets are involved in a collision and there are enough retransmissions containing the same packets. A collision is treated as a linear equation over the involved packets, which can be recovered if the resulting system of linear equations has a unique solution. The asymptotic optimality of ANC in high SNR regimes was recently proved in [21], and its asymptotic optimality in terms of degrees of freedom was shown in [22].

In this work we look at the idea of decoding a collided signal from a different perspective. Extending the hardness results presented in [7], we combine the SINR model with successive interference cancellation capability and analyze the centralized problem of scheduling wireless requests between nodes arbitrarily distributed in the Euclidean space. We propose an algorithm that produces correct and short schedules in this model.

\section{MODEL}

The input to the link scheduling problem is a set of links $L=l_{1}, \ldots, l_{n}$, where each link $l_{x}$ represents a communication request from a sender $s_{x}$ to a receiver $r_{x}$. We assume that nodes live in Euclidean plane. The distance between two nodes $s_{x}, r_{y}$ is denoted by $d_{x y}=d\left(s_{x}, r_{y}\right)$. The length of a link $l_{x}$ is denoted by $d_{x x}$. The received power $P_{r_{x}}\left(s_{x}\right)$ of a signal 
transmitted by sender $s_{x}$ at receiver $r_{x}$ is defined as

$$
P_{r_{x}}\left(s_{x}\right)=\frac{P}{d_{x x}^{\alpha}},
$$

where $P$ is the transmission power, and $d_{x x}^{-\alpha}$ is the propagation attenuation (link gain). The path-loss exponent $\alpha>2$ is a constant, whose exact value depends on external conditions of the medium, such as humidity, obstacles, etc.

In the physical interference model, a receiver $r_{x}$ successfully decodes a transmission from a sender $s_{x}$ iff

$$
S I N R_{r_{x}}\left(\mathcal{S}_{t}\right)=\frac{P_{r_{x}}\left(s_{x}\right)}{\sum_{s_{y} \in \mathcal{S}_{t} s_{y} \neq s_{x}} P_{r_{x}}\left(s_{y}\right)+N} \geq \beta,
$$

where $\mathcal{S}_{t}$ is the set of nodes concurrently transmitting in time-slot $t$, and $\beta$ is the minimum signal-to-interference-plusnoise-ratio (SINR) required for a successful message decoding. Typically, it is assumed that $\beta>1$.

The objective of the Scheduling problem is to compute a minimum-length schedule $\mathcal{S}=\left\{\mathcal{S}_{1} \ldots \mathcal{S}_{T}\right\}$ of size $T$, such that all links in every time slot $\mathcal{S}_{t} \in \mathcal{S}$ can be scheduled successfully according to inequality (1).

We consider the case where all nodes transmit with the same power level $P$, i.e., we assume a uniform power assignment scheme [23].

In order to capture the capability of decoding several signals from a collision, we work with a new definition of a successful transmission, which we introduce below.

\section{A. Successive Interference Cancellation}

Successive interference cancellation is based on the fact that interfering signals, unlike noise, obey a certain structure, determined by the data being transmitted. The idea is to exploit this structured nature in order to decode several collided signals. Firstly, the basic SINR model is used to decode the strongest signal received in a collision, and then the bits decoded from the signal are used to generate an approximated model used to subtract its contribution from the overall interference of the collided signal. As described in [6], a typical receiver would perform the following steps:

1) Detect a collision, by scanning for strong amplitude variations in the incoming signal;

2) Decode the strongest signal using the inequality 1;

3) Use the decoded digital data from (2) and the properties of the physical layer standard, e.g. 802.15.4, to develop an approximated model for the received signal;

4) Use the model developed in (3) to cancel out the strongest signal from the overall interference by iterating through the data and aligning the phase of the model and the received samples;

5) Iterate to decode the remaining packets.

In order to model successive interference cancellation, we assume that a receiver $r$ is able to decode several signals simultaneously, provided that these signals differ in strength significantly.

Consider a set of concurrently scheduled links $\mathcal{S}_{t}$, and a subset of $k$ signals sorted in decreasing order of power received at a node $r: \Upsilon=\left\{P_{r}\left(s_{1}\right), P_{r}\left(s_{2}\right), \cdots, P_{r}\left(s_{k}\right)\right\}$. We assume that the receiver $r$ is able to decode all $k$ signals in $\Upsilon$ if and only if the following condition holds $\forall x \in\{1, \cdots, k\}$ :

$$
\frac{P_{r}\left(s_{x}\right)}{\sum_{\substack{s_{y} \in \mathcal{S}_{t}, P_{r}\left(s_{y}\right) \in \Upsilon, P_{r}\left(s_{y}\right)<P_{r}\left(s_{x}\right)}} P_{r}\left(s_{y}\right)+\sum_{\substack{s_{z} \in \mathcal{S}_{t} \\ P_{r}\left(s_{z}\right) \notin \Upsilon}} P_{r}\left(s_{z}\right)+N} \geq \beta,
$$

where the first component of the denominator is the accumulated interference caused by transmissions in $\Upsilon$, which have weaker power level than $P_{r}\left(s_{x}\right)$; the second component of the denominator is the accumulated interference of all other concurrent transmissions in the network, which are not in $\Upsilon$; $N$ is the ambient noise; and $\beta$ is the minimum SINR threshold.

The idea is that, one by one, each signal $P_{r}\left(s_{x}\right) \in \Upsilon$ can be filtered out from the accumulated interference, provided that the SINR between this signal and the remaining interference is above the threshold $\beta$. The key point here is that a receiver $r$ is able to decode not only the strongest signal, as in the traditional physical interference model, but also a relatively weak signal, provided that each of the stronger signals has been filtered out. Therefore, a signal $P_{r}\left(s_{x}\right)$ can be correctly decoded if and only if all concurrently scheduled stronger signals $\left(P_{r}\left(s_{y}\right)\right)$ obey the following constraints:

$$
\begin{gathered}
\frac{P_{r}\left(s_{y}\right)}{P_{r}\left(s_{x}\right)+\sum \underset{\substack{s_{z} \in \mathcal{S}_{t} \\
s_{z} \neq s_{x} \\
P_{r}\left(s_{z}\right)<P_{r}\left(s_{y}\right)}}{P_{r}\left(s_{z}\right)+N}} \geq \beta, \quad \text { and } \\
\forall s_{y} \in \mathcal{S}_{t}, \text { where } P_{r}\left(s_{y}\right)>P_{r}\left(s_{x}\right), \\
\frac{P_{r}\left(s_{x}\right)}{\sum_{\substack{s_{z} \in \mathcal{S}_{t} \\
P_{r}\left(s_{z}\right)<P_{r}\left(s_{x}\right)}} P_{r}\left(s_{z}\right)+N} \geq \beta .
\end{gathered}
$$

The objective of the Scheduling with Successive Interference Cancellation problem (SSIC) is to compute a minimum-length schedule $\mathcal{S}=\left\{\mathcal{S}_{1} \ldots \mathcal{S}_{T}\right\}$ of size $T$, such that all links in every time slot $\mathcal{S}_{t} \in \mathcal{S}$ can be scheduled successfully according to inequality (2), or equivalently, inequalities (3) and (4).

\section{Algorithm FOR SSIC}

In this section we present a scheduling algorithm that exploits successive interference cancellation in the physical interference model. The algorithm greedily schedules links, checking for successive interference cancellation opportunities at each step. The result is a schedule of length $T$, where in each time slot all transmissions can be decoded successfully according to equation (2). Note that we do not provide approximation guarantees for this algorithm, i.e., we do not know how well it performs in comparison to an optimal solution to the SSIC problem. However, in Section V, we compare its performance to scheduling algorithms that do not employ interference cancellation techniques through simulations, and observe a significant improvement in throughput.

We start by defining a function $\operatorname{SSIC}\left(r, \Upsilon, I, \beta^{\prime}\right)$, which returns true iff a receiver $r$ is able to decode all signals in a given set $\Upsilon$ with SINR threshold $\beta^{\prime}$. More precisely, given a set of $k$ signals (sorted in decreasing order of power 
received by $r) \Upsilon=\left\{P_{r}\left(s_{1}\right), P_{r}\left(s_{2}\right), \cdots, P_{r}\left(s_{k}\right)\right\}$, a set of all other concurrent signals $I$, and an SINR threshold $\beta^{\prime}$, $S S I C\left(r, \Upsilon, I, \beta^{\prime}\right)=$ true iff the following condition holds $\forall x \in\{1, \cdots, k\}$ :

$$
\frac{P_{r}\left(s_{x}\right)}{\sum_{\substack{s_{y} \in \mathcal{S}_{t}, P_{r}\left(s_{y}\right) \in \Upsilon, P_{r}\left(s_{y}\right)<P_{r}\left(s_{x}\right)}} P_{r}\left(s_{y}\right)+\sum_{P_{r}\left(s_{z}\right) \in I} P_{r}\left(s_{z}\right)+N} \geq \beta^{\prime} .
$$

Algorithm 1 starts by setting two constants: $\beta^{\prime}=3 \beta / 2$, a slightly higher SINR threshold than the original $\beta$; and $c$, a constant defined in (6). The algorithm schedules links in increasing order of their length. Once a link $l_{x}$ is selected to be scheduled in time slot $t$ (line 8 ), some of the remaining links $l_{y}$ (those that have not been scheduled yet) are eliminated from the current time slot (and put into set $D$ ) in two steps. To do that, the signals which have already been scheduled in this time-slot $\left(l_{i} \in \mathcal{S}_{t}\right)$ are divided into two subsets: $\Upsilon$, containing signals from senders located within distance $d_{y y}$ of receiver $r_{y}$ (line 10), and $I$, containing signals from the remaining senders in $\mathcal{S}_{t}$ (line 11). In the first elimination step (line 12), all links $l_{y}$ that do not meet the decoding condition $S S I C\left(r_{y}, \Upsilon, I, \beta^{\prime}\right)$ and have an $S I N R_{r_{y}}\left(\mathcal{S}_{t}\right)$ (ratio of signal to the interference from senders in $\mathcal{S}_{t}$, plus noise) lower than $\beta^{\prime}$ are removed. In the second elimination step (line 13), all links whose senders are within distance $c \cdot d_{x x}$ from receiver $r_{x}$ are removed. This process is repeated until all links have been either scheduled in time-slot $t$ or deleted. The whole process is repeated using the deleted links as input, until all links have been scheduled.

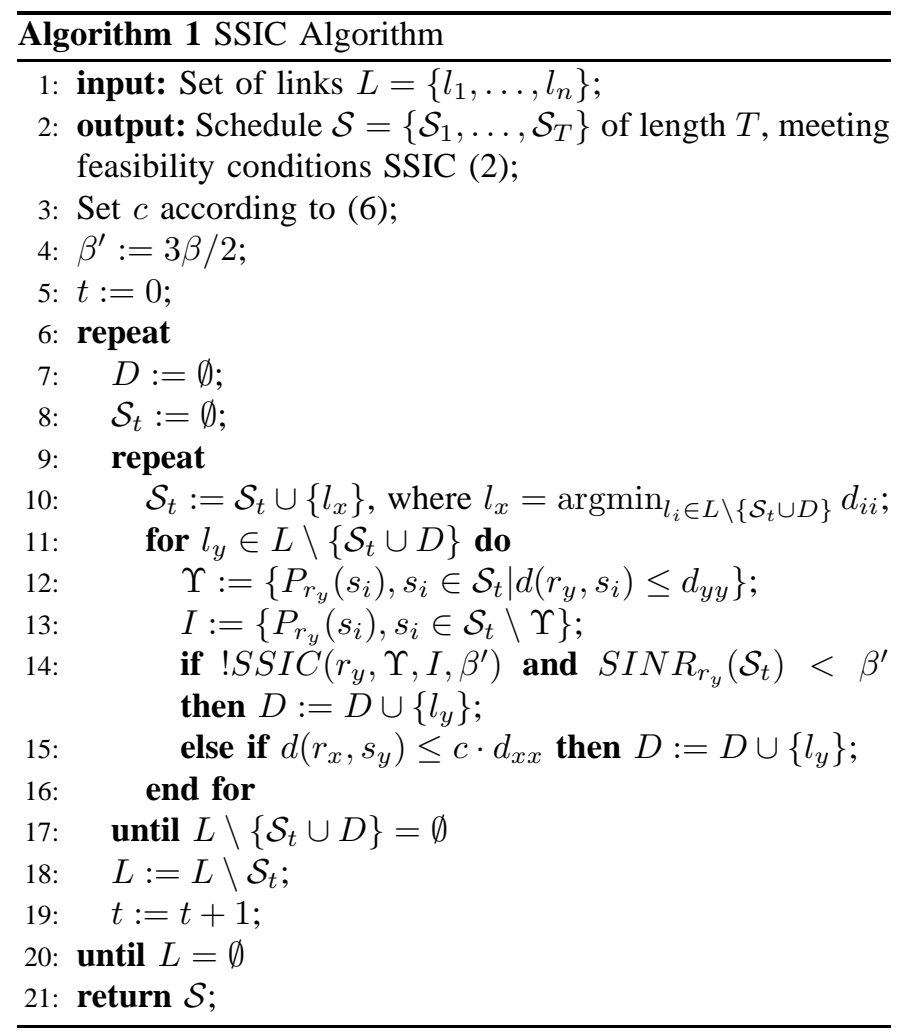

In the following theorem we prove that the schedule $\mathcal{S}$ obtained by Algorithm 1 is correct, i.e., all selected links can be scheduled concurrently without collisions using successive interference cancellation.

Theorem 4.1: Algorithm 1 produces a valid schedule according to SSIC feasibility conditions, defined in (2).

Proof: Consider a time-slot $t$ and an arbitrary link $l_{x}$ scheduled in $\mathcal{S}_{t}$. Let $S_{x}^{-}$be the set of links shorter than $l_{x}$, i.e., those added to $\mathcal{S}_{t}$ before $l_{x}$, and $S_{x}^{+}$be the set of links longer than $l_{x}$, i.e., those added after $l_{x}$. When a link $l_{x}$ is added to the solution, two conditions hold: (1) the signal from the intended sender $s_{x}$ can be decoded with SINR threshold $\beta^{\prime}=3 \beta / 2$, since $l_{x}$ either satisfies $\operatorname{SSIC}\left(r_{x}, \Upsilon, S_{x}^{-} \backslash \Upsilon, \beta^{\prime}\right)$ or $S I N R_{r_{x}}\left(S_{x}^{-}\right) \geq b^{\prime}$, where $\Upsilon=\left\{P_{r_{x}}\left(s_{i}\right)\left|s_{i} \in \mathcal{S}_{t}\right| d\left(s_{i}, r_{x}\right) \leq\right.$ $\left.d_{x x}\right\}$; and (2) senders in $S_{x}^{+}$are located outside the disc of radius $c \cdot d_{x x}$. It remains to show that the additional interference from $S_{x}^{+}$is small enough to allow the signal from $s_{x}$ to be decoded with SINR threshold $\beta$. We need to show that either $\operatorname{SSIC}\left(r_{x}, \Upsilon,\left\{S_{x}^{-} \backslash \Upsilon\right\} \cup S_{x}^{+}, \beta\right)=$ true or $S I N R_{r_{x}}\left(S_{x}^{-} \cup S_{x}^{+}\right) \geq \beta$, i.e.:

- $\operatorname{SSIC}\left(r_{x}, \Upsilon, \mathcal{S}_{t} \backslash \Upsilon, \beta\right)$ or

- $S I N R_{r_{x}}\left(\mathcal{S}_{t} \backslash s_{x}\right) \geq \beta$.

In order to bound the interference from $S_{x}^{+}$we use the fact that, by the second elimination criterion of the algorithm, discs of radius $c \cdot d_{j j}$ around each receiver $r_{j} \in S_{x}^{+}$do not contain any sender $s_{z} \neq s_{j}$. Using this fact and the triangular inequality, we can lower bound the distance between any two senders $\left(s_{j}, s_{z}\right) \in S_{x}^{+}$as $d\left(s_{j}, s_{z}\right) \geq d\left(r_{j}, s_{z}\right)-d_{j j} \geq c$. $d_{j j}-d_{j j}=d_{j j}(c-1) \geq d_{x x}(c-1)$. Therefore, discs $D_{j}$ of radius $d_{x x}(c-1) / 2$ around senders in $S_{x}^{+}$do not intersect.

We partition the space into concentric rings Ring $_{k}$ of width $c \cdot d_{x x}$ around the receiver $r_{x}$. Each ring $\operatorname{Ring}_{k}$ contains all senders $s_{j} \in S_{x}^{+}$, for which $k\left(c \cdot d_{x x}\right) \leq d\left(s_{j}, r_{x}\right) \leq(k+1)(c$. $\left.d_{x x}\right)$. We know that the first ring Ring $_{0}$ does not contain any sender. Consider all senders $s_{y} \in$ Ring $_{k}$ for some integer $k>0$. All discs of radius $d_{x x}(c-1) / 2$ around each $s_{j}$ must be located entirely in an extended ring Ring $_{k}$ of area

$$
\begin{aligned}
A\left(\text { Ring }_{k}\right)= & {\left[\left(d_{x x}(k+1) c+d_{x x}(c-1) / 2\right)^{2}-\right.} \\
& \left.\left(d_{x x} k c-d_{x x}(c-1) / 2\right)^{2}\right] \pi \\
< & (2 k+1) d_{x x}^{2} 2 c^{2} \pi .
\end{aligned}
$$

Since discs of area $A\left(D_{y}\right) \geq\left(d_{x x}(c-1) / 2\right)^{2} \pi$ around senders in $S_{x}^{+}$do not intersect, and the minimum distance between $r_{x}$ and $s_{y} \in \operatorname{Ring}_{k}, k>0$ is $k\left(c \cdot d_{x x}\right)$, we can use an area argument to bound the number of senders inside each ring. The total interference coming from ring $\operatorname{Ring}_{k}, k \geq 1$ is then bounded by

$$
\begin{aligned}
I_{r_{x}}\left(\text { Ring }_{k}\right) & \leq \sum_{s_{y} \in \text { Ring }_{k}} I_{r_{x}}\left(s_{y}\right) \\
& \leq \frac{A\left(\text { Ring }_{k}\right)}{A\left(D_{y}\right)} \frac{P}{\left(k c d_{x x}\right)^{\alpha}} \\
& \leq \frac{(2 k+1) P 2^{3} c^{2}}{k^{\alpha} d_{x x}^{\alpha} c^{\alpha}(c-1)^{2}} \\
& \leq \frac{1}{k^{(\alpha-1)}} \frac{P 2^{5} 3}{d_{x x}^{\alpha} c^{(\alpha)}}
\end{aligned}
$$


where the last inequality holds since $k \geq 1 \Rightarrow 2 k+1 \leq 3 k$ and $c \geq 2 \Rightarrow(c-1) \geq c / 2$. Summing up the interferences over all rings yields

$$
\begin{aligned}
I_{r_{x}}\left(S_{x}^{+}\right) & <\sum_{k=1}^{\infty} I_{r_{x}}\left(\text { Ring }_{k}\right) \\
& \leq \sum_{k=1}^{\infty} \frac{1}{k^{\alpha-1}} \frac{P 2^{5} 3}{d_{x x}^{\alpha} c^{(\alpha)}} \\
& <\frac{\alpha-1}{\alpha-2} \frac{P 2^{5} 3}{d_{x x}^{\alpha} c^{(\alpha)}} \\
& \leq \frac{P_{r_{x}}\left(s_{x}\right)}{3 \beta}
\end{aligned}
$$

where the last two inequalities hold since $\alpha>2$ and $c$ is defined as follows

$$
c=\max \left(2,\left(2^{5} 3^{2} \beta \frac{\alpha-1}{\alpha-2}\right)^{\frac{1}{\alpha}}\right) .
$$

If we define $\Upsilon_{i}^{+}$to be the set of signals in $\Upsilon$ coming from senders located closer to $r_{x}$ than $s_{i}$, we know that, since $\operatorname{SSIC}\left(r_{x}, \Upsilon, S_{x}^{-}, \beta^{\prime}\right)=$ true or $\operatorname{SIN}_{r_{x}}\left(S_{x}^{-}\right) \geq \beta^{\prime}$, the following bounds on interference hold:

$$
\begin{aligned}
I_{r_{x}}\left(S_{x}^{-} \backslash \Upsilon_{i}^{+}\right)+N & \leq \frac{P_{r_{x}}\left(s_{i}\right)}{\beta^{\prime}} \\
& \leq \frac{2 P_{r_{x}}\left(s_{i}\right)}{3 \beta}, \forall P_{r_{x}}\left(s_{i}\right) \in \Upsilon,
\end{aligned}
$$

in case successive interference cancellation is used, and

$$
I_{r_{x}}\left(S_{x}^{-}\right)+N \leq \frac{P_{r_{x}}\left(s_{x}\right)}{\beta^{\prime}}=\frac{2 P_{r_{x}}\left(s_{x}\right)}{3 \beta},
$$

in case no interference cancellation is performed. In both cases, by using the bound (5) on $I_{r_{x}}\left(S_{x}^{+}\right)$(and the fact that $\left.P_{r_{x}}\left(s_{i}\right) \geq P_{r_{x}}\left(s_{x}\right), \forall P_{r_{x}}\left(s_{i}\right) \in \Upsilon\right)$, we obtain

$$
\begin{aligned}
I_{r_{x}}\left(\left\{S_{x}^{-} \backslash \Upsilon_{i}^{+}\right\} \cup S_{x}^{+}\right)+N & \leq \frac{2 P_{r_{x}}\left(s_{i}\right)}{3 \beta}+\frac{P_{r_{x}}\left(s_{x}\right)}{3 \beta} \\
& \leq \frac{P_{r_{x}}\left(s_{i}\right)}{\beta}, \forall P_{r_{x}}\left(s_{i}\right) \in \Upsilon .
\end{aligned}
$$

$\Rightarrow \operatorname{SSIC}\left(r_{x}, \Upsilon, \mathcal{S}_{t} \backslash \Upsilon, \beta\right)$ for encoded transmissions, and

$$
\begin{aligned}
I_{r_{x}}\left(S_{x}^{-} \cup S_{x}^{+}\right)+N & \leq \frac{2 P_{r_{x}}\left(s_{x}\right)}{3 \beta}+\frac{P_{r_{x}}\left(s_{x}\right)}{3 \beta} \\
& \leq \frac{P_{r_{x}}\left(s_{x}\right)}{\beta} .
\end{aligned}
$$

$\Rightarrow S I N R_{r_{x}}\left(\mathcal{S}_{t} \backslash s_{x}\right) \geq \beta$ for non-encoded transmissions. This completes the proof.

\section{Simulation Results}

In this section we present some simulation results to illustrate the gain in throughput obtained by using successive interference cancellation. We generated a topology, where nodes are distributed on a square field of size $W=1000$, and links have different levels of variance in length. More precisely, $n_{C}$ length classes were defined, such that the link length $l_{k}$ in each class $c_{k}, 1 \leq k \leq n_{C}$ is uniformly distributed between $l_{\max }=W / 2^{k}$ and $l_{\min }=W / 2^{k+1}+W / 2^{k+2}$. In each length class, $n / n_{C}$ receiver nodes were distributed uniformly at random in the deployment field, and the respective senders were positioned uniformly at random at distance $l_{k}$ from their intended receivers. With high-link-length-diversity topologies we tried to simulate scenarios, where more interference cancellation opportunities would arise.

We compare the performance of Algorithm 1 to the performance of three scheduling algorithms without coding: GreedyPhysical (proposed in [24]), ApproxDiversity (proposed in [1]), and ApproxLogN (proposed in [8]). As the SSIC Algorithm, all these algorithms are polynomial in time and are specifically designed for the SINR model.

In all experiments, the number of simulations was chosen large enough to obtain sufficiently small confidence intervals.
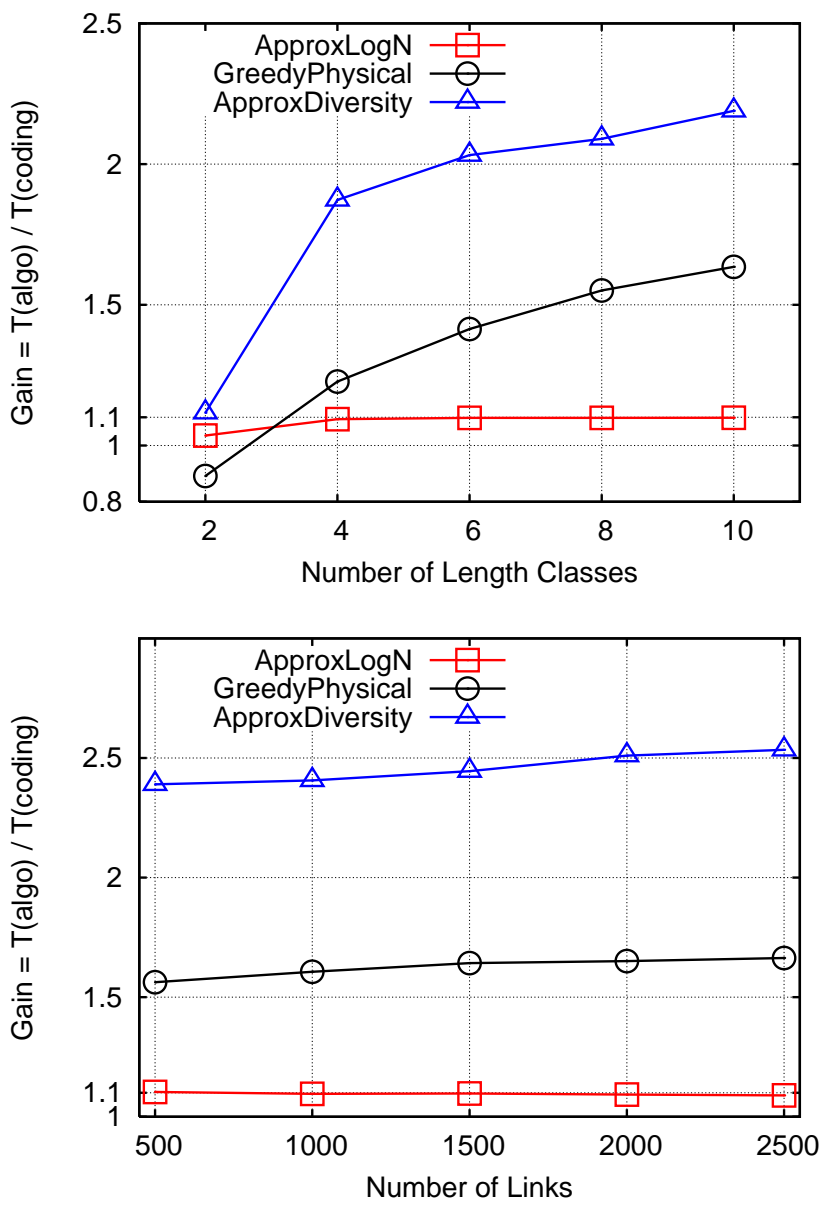

Fig. 1. Gain obtained with SSIC. $\left(n=1000\right.$ when variable $n_{C}, n_{C}=10$ when variable $n, \alpha=5, \beta=1.2$ )

Firstly, we analyze the size of the obtained schedule as a function of the number of length classes (see Figure 1(a)). It can be seen that the more diverse the link lengths, the more interference cancellation opportunities exist in the network, and the higher the gain of the successive interference cancellation approach relative to non-interference cancellation 

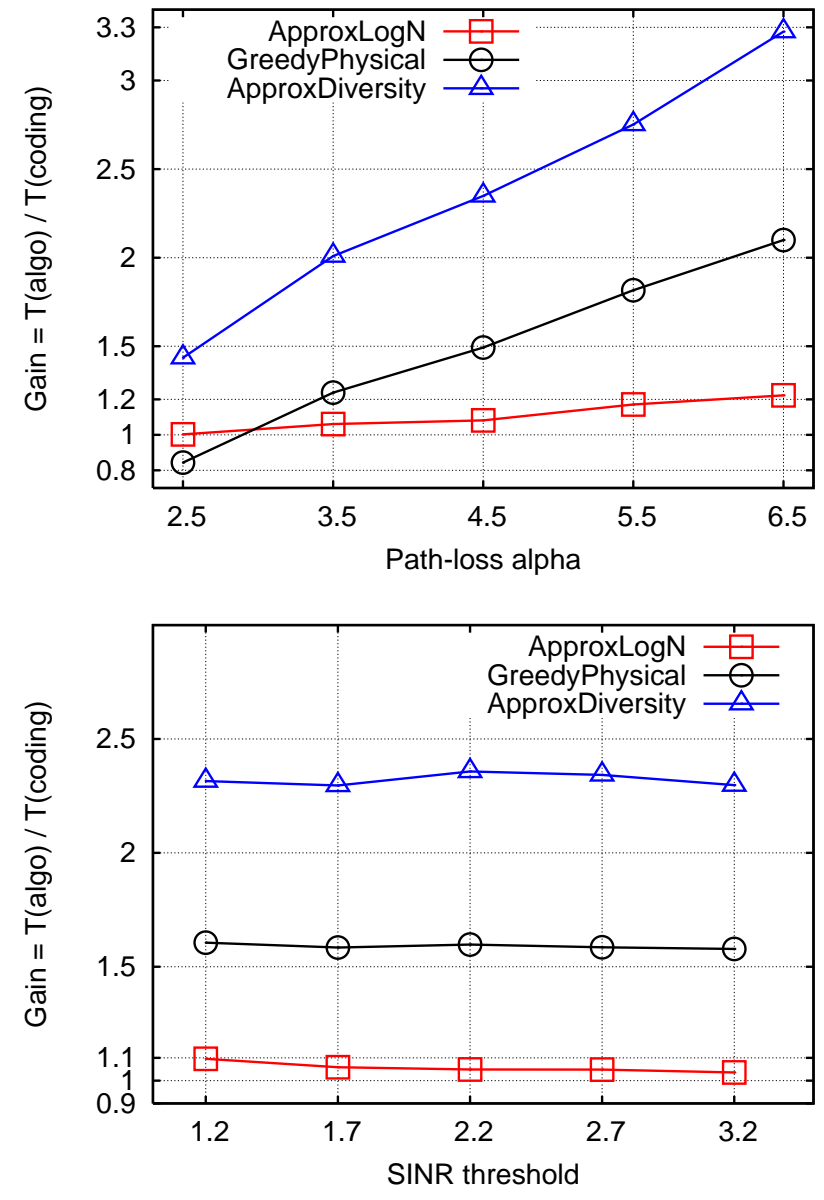

Fig. 2. Influence of SINR parameters. $\left(n=1000, n_{C}=10\right)$

scheduling algorithms. In Figure 1(b) we analyze the influence of the total number of nodes on the relative performance of the algorithms. Since the number of length classes is maintained constant, the number of successive interference cancellation opportunities does not significantly change. Therefore, the gain in throughput due to network coding does not vary much with varying network density. In Figure 2(a) we analyze the impact of the path-loss exponent $\alpha$. It can be observed that when $\alpha<3$, the GreedyPhysical algorithm achieves slightly better performance than the SSIC algorithm. This can be explained by the fact that the second elimination step of Algorithm 1 depends on the constant $c$, defined in (6), which increases sharply when $\alpha$ approaches 2 . For higher values of $\alpha$, however, the successive interference cancellation approach becomes increasingly more efficient. In Figure 2(b) we analyze the impact of the SINR threshold $\beta$. It can be seen that the value of $\beta$ does not influence the performances of the algorithms, which is expected, given that $\beta$ is just a ratio. In Figures 1(a) through 2(b), it can be observed that the throughput gain of coding is the smallest relative to algorithm ApproxLogN. This is due to the fact that ApproxLogN outperforms the other algorithms. Nevertheless, the coding approach achieves gains that vary from $3.5 \%$ (when $n_{C}=2$ ) up to $10 \%$ (when
$n_{C}=10$ ) and $20 \%$ (when $n_{C}=10$ and $\alpha=6$ ).

Overall, the simulation results showed that the gain of successive interference cancellation depends both on the topology of the network and on the SINR parameters. The more interference cancellation opportunities a network topology generates, the more explicit the gain of the SSIC algorithm over traditional scheduling algorithms is. In particular, since network topologies with high link length diversity exhibit stronger power variation between simultaneous packets at the receivers, they induce higher throughput gains due to interference cancellation.

\section{CONCLUSION}

In this work we wanted to obtain an efficient algorithm for scheduling wireless links in the physical interference model with successive interference cancellation capability. Given that interference cancellation changes the definition of a successful transmission, allowing a receiver to decode several messages simultaneously, it is interesting to analyze whether significantly shorter schedules can be obtained for nodes arbitrarily distributed in the Euclidean space.

We proposed a scheduling algorithm that explores interference cancellation opportunities in the network. We showed through simulations that better throughput can be obtained in certain network topologies. In particular, the stronger the signal power variation between simultaneous packets at the receivers in a network topology, the higher are the induced throughput gains due to successive interference cancellation.

\section{REFERENCES}

[1] O. Goussevskaia, Y. A. Oswald, and R. Wattenhofer, "Complexity in Geometric SINR," in ACM International Symposium on Mobile Ad Hoc Networking and Computing (MOBIHOC), September 2007.

[2] J. Hamkins, "An analytic technique to separate cochannel fm signals," IEEE Transactions on Communications, vol. 48, no. 4, pp. 543-546, 2000.

[3] S. Katti, S. Gollakota, and D. Katabi, "Embracing wireless interference: analog network coding," in Proceedings of the ACM SIGCOMM conference. ACM Press, 2007, pp. 397-408.

[4] S. Yoon, "Contrabass: concurrent transmissions without coordination," in Proceedings of the ACM SIGCOMM conference. New York, NY, USA: ACM, 2010, pp. 403-404.

[5] H. Bölcskei, R. U. Nabar, O. Oyman, and A. J. Paulraj, "Capacity scaling laws in MIMO relay networks," IEEE Transactions on Wireless Communications, vol. 5, no. 6, pp. 1433-1444, Jun. 2006.

[6] D. Halperin, T. Anderson, and D. Wetherall, "Taking the sting out of carrier sense: interference cancellation for wireless LANs," in Proceedings of the 14th ACM international conference on Mobile computing and networking (Mobicom). New York, NY, USA: ACM, 2008, pp. 339-350.

[7] O. Goussevskaia and R. Wattenhofer, "Complexity of Scheduling with Analog Network Coding," in ACM International Workshop on Foundations of Wireless Ad Hoc and Sensor Networking and Computing (FOWANC), May 2008.

[8] O. Goussevskaia, M. Halldorsson, R. Wattenhofer, and E. Welzl, "Capacity of Arbitrary Wireless Networks," in 28th Annual IEEE Conference on Computer Communications (INFOCOM), April 2009.

[9] T. Kesselheim, "A Constant-Factor Approximation for Wireless Capacity Maximization with Power Control in the SINR Model," in Proceedings of the 22nd ACM-SIAM Symposium on Discrete Algorithms (SODA), January 2011.

[10] O. Goussevskaia, Y. A. Pignolet, and R. Wattenhofer, "Efficiency of Wireless Networks: Approximation Algorithms for the Physical Interference Model," in Foundations and Trends in Networking, November 2010 . 
[11] J. Hamkins, "Joint viterbi algorithm to separate cochannel fm signals," in IEEE Int. Conf. Acoustics, Speech, Signal Processing, 1998.

[12] S. Verdu, Multiuser Detection. Cambridge University Press, 1998.

[13] J. Andrews, "Interference cancellation for cellular systems: a contemporary overview," Wireless Communications, IEEE, vol. 12, no. 2, pp. 19 - 29, april 2005.

[14] J. Hou, J. Smee, H. Pfister, and S. Tomasin, "Implementing interference cancellation to increase the ev-do rev a reverse link capacity," Соттиnications Magazine, IEEE, vol. 44, no. 2, pp. 58 - 64, feb. 2006.

[15] D. Halperin, J. Ammer, T. Anderson, and D. Wetherall, "Interference cancellation: better receivers for a new wireless MAC," in Proceedings of the 6th ACM workshop on hot topics in networks (Hotnets), 2007.

[16] S. Gollakota, S. D. Perli, and D. Katabi, "Interference alignment and cancellation," in Proceedings of the ACM SIGCOMM conference. New York, NY, USA: ACM, 2009, pp. 159-170.

[17] E. Gelal, K. Pelechrinis, T.-S. Kim, I. Broustis, S. V. Krishnamurthy, and B. Rao, "Topology control for effective interference cancellation in multi-user mimo networks," in Proceedings of the 29th conference on Information communications, ser. INFOCOM'10. Piscataway, NJ, USA: IEEE Press, 2010, pp. 2357-2365. [Online]. Available: http://dl.acm.org/citation.cfm?id=1833515.1833824

[18] C. Avin, A. Cohen, Y. Haddad, E. Kantor, Z. Lotker, M. Parter, and D. Peleg, "Sinr diagram with interference cancellation," in Proceedings of the Twenty-Third Annual ACM-SIAM Symposium on Discrete Algorithms, ser. SODA '12. SIAM, 2012, pp. 502-515. [Online]. Available: http://dl.acm.org/citation.cfm?id=2095116.2095159

[19] S. Zhang, S. C. Liew, and P. P. Lam, "Hot topic: physical-layer network coding," in MOBICOM, 2006, pp. 358-365.

[20] S. Gollakota and D. Katabi, "Zigzag decoding: combating hidden terminals in wireless networks," in Proceedings of the ACM SIGCOMM conference. New York, NY, USA: ACM, 2008, pp. 159-170.

[21] I. Maric, A. Goldsmith, and M. Medard, "Analog network coding in the high-snr regime," in Proceedings of the IEEE Wireless Network Coding Workshop (WINC), 2010.

[22] S. Borade, L. Zheng, and R. Gallager, "Amplify-and-forward in wireless relay networks: Rate, diversity, and network size," Information Theory, IEEE Transactions on, vol. 53, no. 10, pp. 3302 -3318, oct. 2007.

[23] P. Gupta and P. R. Kumar, "Critical Power for Asymptotic Connectivity in Wireless Networks," Stochastic Analysis, Control, Optimization and Applications, pp. 547-566, 1998.

[24] G. Brar, D. Blough, and P. Santi, "Computationally Efficient Scheduling with the Physical Interference Model for Throughput Improvement in Wireless Mesh Networks," in Proc. of the $12^{\text {th }}$ International Conference on Mobile Computing and Networking (MOBICOM), 2006. 